\title{
Turismo y aplicaciones móviles. Preferencias de turistas y prestadores de servicios en el cantón Tena, Napo, Ecuador
}

\author{
Marco Andrés Urvina Alejandro* Xavier Bolìvar Lastra-Bravo** \\ Cecilia Jaramillo-Moreno**** \\ Universidad Central del Ecuador (Ecuador)
}

\begin{abstract}
Resumen: Los teléfonos inteligentes, y sus aplicaciones, han transformado no solo la manera de comunicación, sino también cómo se viaja. Por ello, se analizó su papel actual en el sector turístico de Tena, Ecuador. Primero, se realizó una investigación exploratoria en la plataforma digital Google Play para identificar las aplicaciones de turismo existentes. Posteriormente, se encuestó a visitantes sobre su preferencia de uso de las aplicaciones en sus viajes. Finalmente, se entrevistó a 140 prestadores de servicios turísticos sobre el uso de aplicaciones. Se identificaron 150 aplicaciones, la mayoría (36) corresponden a Pichincha, y solo 4 aplicaciones a Tena. El visitante prefiere informarse a través de sitios web (67\%), y utiliza redes sociales habitualmente (73\%). El 39\% han utilizado aplicaciones durante su visita, siendo Google Maps la más utilizada. Los prestadores de servicios utilizan redes sociales para promoción (57\%), principalmente Facebook, pero el 69\% desconocen o no utilizan aplicaciones de turismo.
\end{abstract}

Palabras Clave: Tecnologías de la información y la comunicación; Servicios turísticos; Smart travel; Aplicaciones móviles; Ecuador.

Tourism and mobile apps. Tourists and service providers' preferences in Tena, Napo, Ecuador

Abstract: Smarthphones and mobile apps have changed not only human communication, but also travelling habits. For this reason, mobile apps influences in the tourism in Tena, Ecuador were analysed. First, exploratory research was carried ou to identify the mobile apps available on Google Play. Later, a survey of the apps preferred by tourists was applied. Finally, 140 service providers were interviewed about their mobile app use. As result, 150 tourism apps were identified for Ecuador, mainly for Pichincha (36) and only 4 for Tena. Tourists prefer web sites (67\%) as a source of tourism information, use social media frequently (73\%), and 39\% used mobile apps during their visit, with Google Maps the most used. service. Providers use social media for promotion (57\%), mainly Facebook, but $69 \%$ of them do not know how to or do not use mobile tourism apps.

Keywords: Information and communication technology; Tourism services; Smart travel; Mobile apps; Ecuador.

\section{Introducción}

Dado que hoy en día la sociedad se encuentra constantemente conectada con el mundo virtual, que le permite adquirir información de su interés, las TICs se han convertido en un pilar importante para el desarrollo del turismo tanto en el ámbito empresarial como comunicacional. La Organización Mundial de Turismo destaca los cambios experimentados en la gestión, consumo y difusión de información por los avances y la combinación de sistemas informáticos y de comunicación, plataformas digitales, redes sociales, sistemas de posicionamiento global, tecnologías móviles e inalámbricas, etc. (UNWTO, 2018a, 2018b). Siendo el sector turístico uno de los sectores económicos protagonista de estos cambios, donde las TICs se han convertido en una herramienta indispensable y útil para las distintas actividades de gestión, logística, distribución y marketing. Por tanto, las TICs juegan un papel muy importante,

\footnotetext{
* Universidad Central del Ecuador (Ecuador); E-mail: maurvina@uce.edu.ec; https://orcid.org/0000-0002-0507-2690

** Universidad Central del Ecuador (Ecuador); E-mail: xblastra@uce.edu.ec; https://orcid.org/0000-0001-6167-6942

**** Universidad Central del Ecuador (Ecuador); E-mail: bcjaramillo@uce.edu.ec; https://orcid.org/0000-0001-6989-9999
} 
pudiendo convertirse en un factor estratégico para el éxito empresarial (Cerezo Medina \& Guevara Plaza, 2015), principalmente dado la proliferación de sitios webs y aplicaciones móviles para promocionar y proveer información de los destinos turísticos. En definitiva, las TICs se han convertido en el motor principal para que una empresa se posicione en el mercado, que el turista pueda informarse, que el destino turístico pueda darse a conocer y facilitar la estadía del visitante, y, que el marketing digital se constituya en una alternativa ideal para un destino turístico que está en surgimiento.

Dada la importancia de las TICs en el sector turístico, en este artículo se presenta un análisis del uso de herramientas digitales por parte de los turistas que visitan el cantón Tena, provincia de Napo, Ecuador, así como también de los prestadores de servicios. Para ello, se presenta

Los resultados obtenidos permitieron analizar la situación actual en cuanto al uso y manejo de herramientas digitales por parte de los turistas y prestadores de servicios turísticos del cantón. Para ello, primero se realizó un estudio exploratorio de las aplicaciones turísticas en Ecuador que están disponibles en Google Play, plataforma de distribución digital de aplicaciones móviles para los dispositivos con sistema operativo Android. Posteriormente, se describe el perfil del uso de aplicaciones móviles por parte de los turistas que visitan el cantón Tena, para ello se aplicó una encuesta a 382 personas (283 turistas nacionales y 99 turistas extranjeros). Finalmente, se analiza el uso de las TICs por parte de los prestadores de servicios turísticos del Tena, para ello se entrevistó a 140 gerentes y empleados de empresas de alojamiento, alimentos y bebidas, diversión nocturna, centros de turismo y agencias de viajes.

\subsection{Sociedad de la Información y herramientas digitales}

La Sociedad de la Información, o sociedad inspirada en el saber de acuerdo con la UNESCO, es producto de las transformaciones y los avances en las Tecnologías de la Información y la Comunicación (TICs) experimentados en los últimos años, caracterizada por facilitar el ofrecer, disponer, compartir y transmitir, información de manera fácil y rápida, superando las barreras físicas y temporales (Cámpora Espí, 2013; MINTEL, 2018b). Las TICs comprenden un conjunto de herramientas y mecanismos tecnológicos que facilitan la información, la comunicación, la creación, la transmisión y el acceso a la información, así como también la construcción y extensión del conocimiento para satisfacer necesidades sociales de distinta índole (Álvarez \& Mayo, 2009; Cámpora Espí, 2013). En este sentido, se entiende que en la actualidad existe una relación más estrecha entre la sociedad y la tecnología, ya que la sociedad contribuye con el intelecto, es decir, personas que cada día están mejorando o creando nuevos avances en tecnología, mientras que la tecnología aporta en el mejoramiento de los niveles de producción, comercialización y comunicación, permitiendo que cada día se facilite el modus vivendi de la sociedad (Urvina Alejandro, 2019).

El internet es la principal herramienta que ha contribuido a la comunicación, convirtiéndose en un fenómeno sociocultural de gran importancia, permitiendo que millones de personas accedan a una mayor cantidad de información (Muñoz Prieto et al., 2013). Ya que las mejoras en infraestructura de radiofrecuencias, avances tecnológicos, mayor accesibilidad para adquirir el servicio, ofertas y promociones, de las operadoras tanto de internet fijo como móvil, han provocado que cada vez aumente el número de usuarios y por supuesto la necesidad de estar conectado con el resto del mundo. El Observatorio TIC del Ministerio de Telecomunicaciones y de la Sociedad de la Información (MINTEL, 2019) señala que a 2017 el 60.68\% de los ecuatorianos usan TICs y que el 94,68\% usan redes sociales en su teléfono, con 9.484 .772 abonados a internet móvil y 2.015.159 a internet fijo en el 2019. A nivel mundial, para el 2025, se prevé que existirán 5.900 millones de suscriptores a internet, lo que equivale al 71\% de la población mundial prevista (Muñoz, 2018).

Una de las herramientas que más ha impactado son los denominados teléfonos inteligentes (smartphones), que han generado grandes cambios y transformaciones en la manera de viajar, generando un modelo integral de viajes inteligentes, ya que a través de estos dispositivos se puede reservar y gestionar un vuelo, comprar online productos y servicios turísticos, obtener información de destinos, planificar el viaje, compartir experiencias durante y después del viaje, etc. (Blanco, 2015; Jacobsen \& Munar, 2012; UNWTO, 2018b). Para ello, se han desarrollado herramientas digitales (blogs, big data, software ágil para la gestión, redes sociales, realidad virtual o aumentada, wiki, aplicaciones móviles - Apps o web Apps, etc.) que han adquirido mayor acogida por parte de los turistas para planificar y disfrutar su viaje. Todo ello ha llevado que el sector turístico proponga e innove la oferta turística, siendo más atractiva, eficiente, inclusiva y sostenible en términos económicos, sociales y ambientales (UNWTO, 2018a).

\subsection{TICs en el sector turístico}

Albacete Sáez \& Herrera Fernández (2012) mencionan que las TICs posibilitan una adecuada gestión de la información y la interacción entre la empresa y el cliente la cual facilita, por un lado, 
que los clientes tengan información más completa y precisa sobre el servicio que pretenden adquirir, y, por otro lado, que las empresas obtengan una mayor información sobre los gustos y necesidades de los clientes para así poder ofrecer unos servicios más ajustados a sus expectativas. Según Andrade Suárez (2012), Cámpora (2013), Segovia Borray et al. (2014) y Grevtsova (2016), las ventajas que se obtienen al implementar las TIC en el sector turístico son:

- Oportunidades para la creación y difusión de la imagen de los destinos turísticos.

- Incrementan y mejoran los flujos de información.

- Ofrecen la posibilidad de obtener e intercambiar grandes cantidades de información muy útil de una manera sencilla y rápida, a través de herramientas tales como las redes sociales, páginas web y blog.

- Generan experiencias únicas y de calidad, con carácter lúdico, al momento de consultar información de un destino.

- Para las empresas la implementación de las TIC mejora la comunicación con los clientes, reduce los costos de operación, reduce barreras en materia de tiempo, mejora el control de las operaciones y genera una buena imagen corporativa.

- Inserción al comercio electrónico para las pequeñas y medianas pymes.

Aramendia-Muneta \& Ollo-López (2013) y Januszewska et al. (2015) destacan que el impacto de las TICs en la competitividad, la productividad, la cuota de mercado y la innovación es muy diferente según el subsector turístico. Identificando que en los subsectores de alojamiento y restauración las TIC tienen un gran impacto positivo en general, mientras que en el subsector de agencias de viajes generan un impacto positivo en la cuota de mercado y la innovación, pero negativo en la competencia y la productividad. Farkhondehzadeh et al. (2013) destacan que el comercio electrónico, asociado a las TICs, ofrece más oportunidades y posibilidades para llegar a un mayor público, intercambio inmediato de información y la realización de transferencias electrónicas, aspectos que en general aumentan la eficiencia económica y abre en mayor medida el mercado de bienes y servicios. En el caso de las empresas turísticas, les permite abrir su oferta a todo el mundo.

Las aplicaciones o Apps de turismo juegan un papel relevante en el auge del uso de teléfonos inteligentes en este sector, dada su alta penetración en el mercado. Sanz Blas et al. (2012) consideran que la aplicación móvil es una herramienta de gestión, donde el usuario tiene la posibilidad de desarrollar determinadas tareas como búsqueda, información, localización, etc. Desde una visión de marketing, Sánchez Jiménez \& Ravina Ripoll (2017) añaden que las aplicaciones móviles generan un valor añadido al cubrir, de manera rápida y personalizada, las necesidades de los consumidores, y al ser un soporte de empresas y destinos para comunicar, publicitarse, impactar, relacionarse, intercambiar y comercializar. En el portal digital Statista, Clement (2020) presenta información sobre descargas de aplicaciones móviles a nivel mundial para 2019, contabilizando un total de 204.000 millones de descargas de aplicaciones móviles. De acuerdo con Google Play Store, en enero de 2018, Facebook, WhatsApp y Google fueron las Apps más descargadas, sin incluir la categoría juegos. Facebook ocupa el primer lugar con más de 130 millones de descargas mensuales en todo el mundo (Aplicación Annie, 2018). En cuanto a ingresos, se estima que para 2020, las aplicaciones móviles generarán ingresos por USD 188.900 millones, a través de tiendas de aplicaciones y publicidad integrada en la aplicación (Aplicación Annie, 2016).

Otro aspecto importante para considerar, y que tiene gran influencia en el uso y manejo de las herramientas digitales, es la edad de los turistas, por los cambios socioculturales específicos que se dan en cada generación. Florido-Benítez et al. (2015) destaca el caso de los segmentos jóvenes, quienes son adeptos a las nuevas tecnologías, son más activos y tienden a estar mejor informados, por lo que obligan a las empresas a innovar constantemente. Molano et al. (2014) consideran tres generaciones que han sido marcadas por el proceso del desarrollo tecnológico:

- Generación X: crecieron junto a la tecnología, creen en "trabajar para vivir", y gozan de amplia movilidad laborar. Nacidos entre 1965 y 1979, "los X" son la primera generación que crece con las computadoras, la tecnología se incorpora a sus vidas.

- Generación del Milenio o Generación Y: nacidos entre 1980 y 2000, son conocidos también como los nativos digitales. Los Millennials representan la nueva fuerza de trabajo global, al menos la mitad de ellos tiene su propio negocio o planea tener uno y están permanentemente conectados a las redes sociales, internet y a los dispositivos móviles que les permite establecer y conectar con nuevas relaciones laborales y profesionales entre sus círculos.

- Generación Z: conforman las personas nacidas en las dos últimas décadas. Está compuesta por adolescentes y jóvenes que han crecido con una notable influencia por internet, las redes sociales, los 
dispositivos móviles. Tienen un permanente contacto con sus dispositivos móviles, debido a la necesidad inherente de estar conectados a las redes y plataformas sociales. A través de estas tienen una estrecha comunicación con sus pares y se enteran sobre lo que está aconteciendo en el resto del mundo.

Por ello, en la actualidad se habla de un turista digital, más autónomo, hiperconectado y cada vez más exigente, ya que espera servicios personalizados y de mayor calidad, cuidadosos de su tiempo y dinero (Bethapudi, 2013; UNWTO, 2018b). Este tipo de turista utiliza los distintos medios digitales como canales válidos para poder recopilar información sobre los destinos turísticos, generar y expresar opiniones y, asimismo, transformar la experiencia de viaje (Blanco, 2015). En este punto, se debe encontrar nuevas fórmulas y estrategias para estar a la vanguardia de este nuevo perfil de turista y para ello se debe estudiar su comportamiento y cómo atraerlo (Andalucía es digital, 2019; Ruiz Gómez et al., 2016). La Junta de Andalucía, en su portal digital "Andalucía es digital", detalla los siguientes tipos de clientes:

- Cliente hiperconectado: emplea nuevas tecnologías, y en especial su teléfono móvil para resolver todos los aspectos relacionados con sus viajes, desde el uso de buscadores especializados en viajes, reservas de vuelos o el check-in en hoteles, pasando por el uso de aplicaciones para localizar sitios de interés o para guiarse en el trayecto (Google Maps para viajes), hasta dejar su opinión o valoración de los servicios turísticos en sus perfiles de redes sociales.

- Cliente informado: el turista digital se informa de todo lo relacionado con su lugar de destino y, lo más importante, consulta en foros y redes sociales especializadas la opinión de otros usuarios sobre hoteles, restaurantes y puntos de interés.

- Cliente que interactúa: tiene relación con el cliente informado, pero debe obtener respuestas y un trato personalizado en los servicios turísticos que va a contratar.

- Cliente que busca nuevas experiencias: las ventajas en la relación entre el turismo y nuevas tecnologías es la posibilidad que ofrecen las TICs al sorprender al visitante, de personalizar su experiencia y los servicios que se le ofrece.

Todos estos cambios tecnológicos han influido en el sector turístico, de manera que la innovación tecnológica es un pilar fundamental en la actualidad para la promoción de un destino turístico, de manera que se pueda llegar a nuevos tipos de clientes, más exigentes y que buscan productos y servicios personalizados. Es así como, el internet se ha transformado en una herramienta fundamental, no sólo para comunicar, entretener o informar, sino que es cada vez más importante a la hora de definir los consumos (Maschini, 2012 , p. 6). El marketing digital es una de las herramientas idóneas para dar a conocer un producto o servicio, tanto a nivel nacional o internacional, que se caracteriza por utilizar herramientas digitales, las cuales permiten que la empresa formule estrategias y mecanismos de comercialización online para llegar mejor al mercado objetivo. A través de las herramientas digitales los usuarios pueden visualizar, disponer de información y, sobre todo, realizar recomendaciones o denuncias de los productos o servicios utilizados. $\mathrm{Al}$ respecto, Ukpabi \& Karjaluoto (2017) señalan que es primordial que los gestores del sector turístico comprendan que no solo se deben usar las herramientas digitales, sino que es importante considerar y comprender el papel del diseño de sitios web, redes sociales y de las aplicaciones móviles.

En la actualidad, los destinos turísticos disponen de un sin número de medios digitales para darse a conocer. Así mismo, la informática y las telecomunicaciones contribuyen al fortalecimiento del destino. Lemos Gomes et al. (2018) señalan a las TICs como vectores de transformación de los destinos turísticos emergentes en consolidados o destinos turísticos inteligentes, de manera que se construyan espacios innovadores, sostenibles y competitivos para los turistas y los residentes. En el ámbito de los medios digitales, los sitios web, las plataformas digitales tales como TripAdvisor, Booking, Trivago, Despegar.com etc., se han popularizado en los últimos años, las cuales facilitan planificar un viaje. Las redes sociales (Facebook, Twitter, Instagram, Youtube) son los medios más populares, ya que los turistas las utilizan antes y durante sus viajes para visualizar información y publicar las actividades realizadas en dicho destino. Mientras que, en el ámbito de las telecomunicaciones, los teléfonos inteligentes determinan la conexión turista-destino, por ello las aplicaciones móviles son las herramientas claves para que el turista encuentre la información adecuada en el lugar de visita.

\section{Metodología}

En primer lugar, se realizó una investigación exploratoria sobre las aplicaciones de turismo en Ecuador disponibles en la plataforma más utilizada: Google Play, entre los meses de mayo a junio de 2018. En 
la búsqueda se utilizaron como palabras claves: "Apps de turismo en Ecuador", "Apps de turismo", "Quito", "Guayaquil”, "Cuenca", "Napo, "Tena”, "Apps Ecuador", "Apps de Galápagos”. De manera que se clasificaron las aplicaciones disponibles a nivel nacional, y se identificaron aquellas disponibles para el cantón Tena y la Provincia del Napo.

Para determinar las características y preferencias de los turistas/usuarios en cuanto a aplicaciones móviles, se procedió a realizar un estudio de mercado, mediante la aplicación de una encuesta a turistas del cantón Tena. Se consultó al turista sobre sus preferencias de uso de Apps móviles, los medios digitales que utiliza para informarse en sus viajes, y el uso de una App de turismo a nivel general o ecuatoriana. Para calcular la muestra se aplicó la fórmula de población finita, con un error del 5\% y una probabilidad del 50\%, considerando un universo de 68.820 turistas que visitaron el cantón durante los meses de enero-agosto de 2017, de acuerdo con el Consolidado de Afluencia de Turistas en el Cantón Tena 2006-2017 (GADM Tena, 2017b). Se obtuvo una muestra de 382 personas a encuestar. La muestra fue segmentada de acuerdo con el origen del turista, 283 nacionales y 99 extranjeros.

Para conocer las preferencias de los prestadores de servicios turísticos se procedió a entrevistar a 140 dueños, gerentes, o empleados de empresas de alojamiento, alimentos y bebidas, diversión nocturna, centros de turismo y agencias de viajes, sobre los medios que utilizan para comercializar y promocionar su establecimiento, así como el uso de Apps de turismo, con ello se obtuvo información relevante que complementó la presente investigación. Este número corresponde al 63\% de los prestadores de servicios incluidos en el Catastro del Cantón Tena 2017 (GADM Tena, 2017a). La muestra fue calculada con la fórmula de población finita, con un universo de 222 establecimientos, y se segmentó de acuerdo con el servicio que brinda: 65 de Alojamiento, 46 de Alimentos y Bebidas, 8 de Diversión Nocturna, 4 Centros de Turismo y 17 Agencias de Viajes.

Tanto el cuestionario aplicado a los turistas como el guion de entrevista a los prestadores de servicios fueron construidos a partir de la revisión de estudios previos y validados por expertos de la Universidad Central del Ecuador.

\section{Resultados}

\subsection{Aplicaciones turísticas en Ecuador disponibles en Google Play}

Como se mencionó anteriormente, en el sector turístico las nuevas tecnologías han ayudado al mejoramiento de la planificación de un viaje. Sin duda, los teléfonos inteligentes se han convertido en la herramienta principal del viajero, que le brinda una variedad de servicios de información para organizar las actividades esenciales del viaje como planificación, reservas de servicios y desplazamientos, así como complementarias dentro del ciclo de viaje, localizar gasolineras, estimar el tiempo de espera de paseos, etc. (Wang \& Fesenmaier, 2010, citado en (Vieira Soares et al., 2017). Por ende, las aplicaciones son un canal adecuado para la gestión de un destino mediante la promoción y un medio adecuado para la comercialización de servicios de las empresas turísticas. Mientras que los teléfonos inteligentes adquieren mayor interés en el mercado y su innovación es constante. Podría decirse que estas dos herramientas son los pilares fundamentales para la nueva forma de hacer turismo.

En Ecuador, tres de cada cuatro personas poseen un teléfono inteligente, en un 90,08\% acceden a tecnología $3 \mathrm{G}+4 \mathrm{G}$, el 94,68\% utiliza redes sociales en sus teléfonos, el 91,98\% internet, y el 76,22\% GPS (MINTEL, 2018a). A nivel internacional, Ecuador se encuentra en el puesto 97 de 176 en el desarrollo de las TICs, mientras que a nivel regional ocupa el puesto ocho, con una diferencia de 2,32 puntos de Uruguay, primero en el ranking. Dado el alto nivel de penetración en el mercado de estos dispositivos, las aplicaciones móviles poseen un alto margen de desarrollo. Esta situación se observó al realizar la investigación exploratoria en Google Play de las aplicaciones turísticas disponibles.

Se identificaron 150 Apps relacionadas con la actividad turística, de las cuales la aplicación Ecuador Travel Guide tiene el mayor ranking de descargas (Tabla 1). Además, se identificaron aplicaciones gestionadas por las entidades públicas como: Guayaquil es mi Destino, Napo Amazonía, Guayaquil Travel, GOUIO, ZAAG, etc. La región Andina sobresale con el mayor número de Apps registradas (65), siendo la provincia de Pichicha la que posee más Apps en la región (36). A nivel local, Quito posee el mayor número de Apps, posiblemente porque es una de las ciudades más visitadas a nivel nacional, es Patrimonio Cultural de la Humanidad, y ha sido galardonada con premios internacionales como los World Travel Awards (WTA), considerados los Oscar de Turismo. Esto ha provocado un desenlace en la elaboración y promoción de aplicaciones móviles de turismo para Ecuador en los últimos años.

Por categorías de Google Play, se determinó que las Apps de viajes representan el 85\%, el idioma español es el más utilizado, seguido del inglés. La aplicación de mayor antigüedad de aplicación es del 
2013, y en 19 de las 24 provincias existen aplicaciones regionales y locales. Por descargas, las Apps con 10.000+ descargas son Ecuador Travel Guide, Guayaquil en tu Mano, Mapas de Ecuador Gratis, MovilizateUIO, Quito App, y con 5.000+ descargas, Cuenca Cultura, Guayaquil es mi Destino, Mapa de Guayaquil Offline, Quito en tú Mano.

El cantón Tena, provincia de Napo cuenta con cuatro App turísticas para promocionar sus destinos a nivel provincial y cantonal, las cuales funcionan online, es decir, requieren conexión a una red inalámbrica o servicio de datos móviles. Esta situación representa un problema para el turista ya que, en algunos sectores, especialmente rurales, no existe una adecuada conectividad, dificultando el uso de las Apps. Dado que las aplicaciones móviles, al ser herramientas multiusos, permiten ofrecer servicios y productos personalizados, de manera que se mejora la experiencia del turista y se maximiza el ocio y disfrute (Florido-Benítez et al., 2015), la importancia de mejorar la conectividad aumenta, y por tanto este tema debería ser considerado como algo prioritario por los gestores locales.

Tabla 1: Base de datos App de viajes, turismo y de mapas y navegación de Ecuador en la plataforma Google Play

\begin{tabular}{|c|c|c|c|c|c|}
\hline Región & Cat $^{1}$ & Nombre & $\mathbf{A n ̃ o}^{2}$ & Idioma $^{3}$ & Descargas \\
\hline \multirow{34}{*}{ Nacional } & $\mathrm{E}$ & WonderfulEcuador_1_2 & 2015 & $\mathrm{I}$ & $100+$ \\
\hline & \multirow{5}{*}{$\mathrm{MyN}$} & Mapa de Ecuador & 2017 & $\mathrm{E}$ & $10+$ \\
\hline & & Go Ec & 2018 & $\mathrm{I}$ & $100+$ \\
\hline & & Mapa de Ecuador offline & 2018 & $\mathrm{E}$ & $500+$ \\
\hline & & Mapas de Ecuador Gratis - Navegación sin Internet & 2018 & $\mathrm{E}$ & $10.000+$ \\
\hline & & Mapas y Navegación GPS sin Internet: Ecuador & 2018 & $\mathrm{E}$ & $100+$ \\
\hline & \multirow{28}{*}{$\mathrm{V}$} & Booking Ecuador Hotels & 2018 & $\mathrm{I}$ & $1+$ \\
\hline & & Club Visita & 2018 & $\mathrm{E}$ & $1.000+$ \\
\hline & & Colourful Ecuador Travels & 2017 & I & $100+$ \\
\hline & & Discover Ecuador & 2016 & E, I & $100+$ \\
\hline & & Ecuador \& Galapagos - FREE Guide & 2015 & I & $1.000+$ \\
\hline & & Ecuador \& Galapagos - Guide & 2015 & I & $100+$ \\
\hline & & Ecuador Hotel Discount & 2016 & I & $500+$ \\
\hline & & Ecuador Offline Travel Guide & 2016 & $\mathrm{E}, \mathrm{I}$ & $1000+$ \\
\hline & & Ecuador Popular Tourist Places and Tourism Guide & 2018 & I & $50+$ \\
\hline & & Ecuador Tourist Places (Guide) & 2017 & $\mathrm{I}$ & $10+$ \\
\hline & & Ecuador Travel & 2016 & E, I & $50+$ \\
\hline & & Ecuador Travel \& Explore & 2017 & $\mathrm{I}$ & $50+$ \\
\hline & & Ecuador Travel Guide & 2016 & $\mathrm{E}, \mathrm{I}$ & $10.000+$ \\
\hline & & Ecuador Travel Guide - RA (no publicada) & 2017 & $\mathrm{E}, \mathrm{I}$ & $1+$ \\
\hline & & Ecuador Travel Guide (no publicada) & 2018 & $\mathrm{E}, \mathrm{I}$ & $10+$ \\
\hline & & Ecuador Wallpaper & 2017 & $\mathrm{E}$ & $50+$ \\
\hline & & Ecuador Wallpaper Travel & 2016 & $\mathrm{E}$ & $500+$ \\
\hline & & Ecuador: Guía & 2018 & $\mathrm{E}, \mathrm{I}$ & $10+$ \\
\hline & & Ecuadoria & 2016 & $\mathrm{I}$ & $5+$ \\
\hline & & EcuApp Travel & 2016 & $\mathrm{E}$ & $5+$ \\
\hline & & EcuaTour & 2017 & $\mathrm{E}$ & $500+$ \\
\hline & & EcuaTour & 2018 & $\mathrm{E}$ & $1.000+$ \\
\hline & & Ecuatur & 2018 & $\mathrm{E}$ & $5+$ \\
\hline & & ETG - Ecuador Travel Guide & 2018 & $\mathrm{E}, \mathrm{I}$ & $10+$ \\
\hline & & GO Ecuador & 2018 & $\mathrm{E}$ & $10+$ \\
\hline & & Hotels Ecuador by tritogo.com & 2016 & $\mathrm{I}$ & $100+$ \\
\hline & & Lugares Turísticos & 2018 & $\mathrm{E}$ & $10+$ \\
\hline & & Mi Ecuador & 2017 & $\mathrm{E}, \mathrm{I}$ & $50+$ \\
\hline
\end{tabular}




\begin{tabular}{|c|c|c|c|c|c|}
\hline Región & Cat $^{1}$ & Nombre & $\mathbf{A n ̃ o}^{2}$ & Idioma $^{3}$ & Descargas \\
\hline & & NIS & 2018 & $\mathrm{E}$ & $500+$ \\
\hline & & Offline Mapa: Ecuador & 2017 & $\mathrm{E}$ & $500+$ \\
\hline & & Rainforest Connection & 2018 & $\mathrm{I}$ & $1.000+$ \\
\hline & & Turismo en Ecuador & 2018 & $\mathrm{E}$ & $50+$ \\
\hline & & Ubica Ecuador & 2018 & $\mathrm{E}$ & $1.000+$ \\
\hline & & Wonderful Ecuador & 2018 & $\mathrm{I}$ & $10+$ \\
\hline & & Áreas Protegidas del Ecuador & 2015 & $\mathrm{E}, \mathrm{I}$ & $1000+$ \\
\hline \multirow{4}{*}{ Azuay } & $\mathrm{MyN}$ & Parque Nacional Cajas & 2018 & $\mathrm{E}$ & $50+$ \\
\hline & NyR & Cuenca Cultura & 2017 & $\mathrm{E}, \mathrm{I}$ & $5.000+$ \\
\hline & \multirow{2}{*}{ V } & Cuenca en tu Mano & 2016 & $\mathrm{E}, \mathrm{I}, \mathrm{P}, \mathrm{F}, \mathrm{It}$ & $1.000+$ \\
\hline & & Guíame Cuenca & 2013 & $\mathrm{E}$ & $500+$ \\
\hline \multirow{3}{*}{ Cañar } & MyN & ZAAG & 2017 & $\mathrm{E}$ & $500+$ \\
\hline & \multirow{2}{*}{$\mathrm{V}$} & Turismo Cañar & 2018 & $\mathrm{E}$ & $10+$ \\
\hline & & Turismo Mancomunidad Cañari & 2017 & $\mathrm{E}, \mathrm{I}$ & $50+$ \\
\hline \multirow{2}{*}{ Chimborazo } & $\mathrm{MyN}$ & TurismoRio & 2017 & $\mathrm{E}$ & $10+$ \\
\hline & $\mathrm{V}$ & Guia Turística de Riobamba & 2016 & E, I & $100+$ \\
\hline \multirow{5}{*}{ Cotopaxi } & \multirow{2}{*}{$\mathrm{E}$} & Cultura Tungurahua & 2018 & $\mathrm{E}$ & $100+$ \\
\hline & & Vive Turismo Cotopaxi & 2018 & $\mathrm{E}$ & $10+$ \\
\hline & \multirow{3}{*}{$\mathrm{V}$} & Mama Negra & 2017 & $\mathrm{E}$ & $10+$ \\
\hline & & Sigchos Turismo & 2016 & $\mathrm{E}$ & $100+$ \\
\hline & & Turismo Municipio Latacunga & 2017 & $\mathrm{E}$ & $10+$ \\
\hline \multirow{2}{*}{ El Oro } & \multirow{2}{*}{$\mathrm{V}$} & Hoteles y Hostales Machala & 2017 & $\mathrm{E}$ & $50+$ \\
\hline & & Machala Contigo & 2016 & $\mathrm{E}$ & $100+$ \\
\hline \multirow{12}{*}{ Galápagos } & \multirow{12}{*}{$\mathrm{V}$} & Galápagos Guía turística en español y mapa & 2018 & $\mathrm{E}$ & $10+$ \\
\hline & & Galapagos Islands Offline Travel Guide & 2018 & $\mathrm{I}$ & $0+$ \\
\hline & & Galapagos Offline Travel Guide (2) & 2017 & I & $10+$ \\
\hline & & Galapagos Tours & 2017 & $\mathrm{I}$ & $50+$ \\
\hline & & Galatrip - Islas Galápagos & 2016 & E, I & $500+$ \\
\hline & & GPS Guía de Productos y Servicios en Galápagos & 2018 & $\begin{array}{c}\text { E, I, P, F, } \\
\quad \text { A, It }\end{array}$ & $10+$ \\
\hline & & INTEMPRE-Galapagos Bipros & 2015 & $\mathrm{I}$ & $10+$ \\
\hline & & Isla Isabela Map & 2015 & $\mathrm{E}$ & $10+$ \\
\hline & & Lindblad Galapagos & 2018 & $\mathrm{I}$ & $10+$ \\
\hline & & Me Gusta Galapagos & 2018 & $\mathrm{I}$ & $10+$ \\
\hline & & Parque Nacional Galápagos Of & 2016 & $\mathrm{E}, \mathrm{I}$ & $1.000+$ \\
\hline & & Stella Maris Galapagos & 2018 & $\mathrm{I}$ & $10+$ \\
\hline \multirow{11}{*}{ Guayas } & \multirow{11}{*}{$\mathrm{V}$} & Conoce Guayaquil & 2017 & $\mathrm{E}$ & $50+$ \\
\hline & & Guayaquil Guía Turística & 2017 & $\mathrm{E}$ & $10+$ \\
\hline & & Guayaquil Ecuador Map & 2017 & $\mathrm{I}$ & $500+$ \\
\hline & & Guayaquil en tu Mano & 2016 & $\begin{array}{c}\mathrm{E}, \mathrm{I}, \mathrm{P}, \mathrm{F} \\
\quad \mathrm{A}, \mathrm{It}\end{array}$ & $10.000+$ \\
\hline & & Guayaquil es mi Destino & 2017 & $\mathrm{E}$ & $5.000+$ \\
\hline & & Guayaquil Guía turística y mapa & 2018 & $\mathrm{E}$ & $10+$ \\
\hline & & Guayaquil Street Map & 2013 & $\mathrm{E}$ & $10+$ \\
\hline & & Guayaquil Travel & 2017 & $\mathrm{E}$ & $50+$ \\
\hline & & Guayaquil: Guía & 2018 & $\mathrm{E}, \mathrm{I}$ & $100+$ \\
\hline & & Mapa de Guayaquil Offline & 2018 & $\mathrm{E}$ & $5.000+$ \\
\hline & & Patrimonios de Guayaquil UG & 2017 & $\mathrm{E}$ & $10+$ \\
\hline
\end{tabular}




\begin{tabular}{|c|c|c|c|c|c|}
\hline Región & Cat $^{1}$ & Nombre & $\mathbf{A n n o}^{2}$ & Idioma $^{3}$ & Descargas \\
\hline \multirow{6}{*}{ Imbabura } & $\mathrm{E}$ & Vive Cotacachi & 2017 & $\mathrm{E}$ & $5+$ \\
\hline & \multirow{5}{*}{$\mathrm{V}$} & Cotacachi Travel & 2018 & $\mathrm{E}$ & $5+$ \\
\hline & & Destino Pimampiro & 2017 & $\mathrm{E}$ & $100+$ \\
\hline & & Otavalo es Turismo & 2018 & $\mathrm{E}$ & $10+$ \\
\hline & & Pablo Arenas Turismo & 2016 & $\mathrm{E}$ & $10+$ \\
\hline & & TourIbarra - Ibarra Turismo & 2016 & E, I & $100+$ \\
\hline \multirow{3}{*}{ Loja } & $\mathrm{H}$ & Agenda Cultural Loja & 2018 & $\mathrm{E}$ & $1.000+$ \\
\hline & \multirow{2}{*}{$\mathrm{V}$} & Loja Turism & 2015 & $\mathrm{E}$ & $50+$ \\
\hline & & Zoológico Loja & 2015 & $\mathrm{E}$ & $100+$ \\
\hline \multirow{3}{*}{ Manabí } & $\mathrm{E}$ & Camino Turistico & 2018 & $\mathrm{E}$ & $500+$ \\
\hline & \multirow{2}{*}{$\mathrm{V}$} & Manabí en tu Mano & 2016 & $\mathrm{E}, \mathrm{I}, \mathrm{P}, \mathrm{F}, \mathrm{A}, \mathrm{It}$ & $1.000+$ \\
\hline & & Manta Es Todo & 2018 & $\mathrm{E}$ & $500+$ \\
\hline \multirow{2}{*}{$\begin{array}{l}\text { Morona } \\
\text { Santiago }\end{array}$} & \multirow{2}{*}{ V } & Morona Santiago es Sangay (no publicada) & 2018 & $\mathrm{E}$ & $10+$ \\
\hline & & Visita Morona Santiago & 2017 & $\mathrm{E}$ & $100+$ \\
\hline \multirow{4}{*}{ Napo } & $\mathrm{MyN}$ & Napo Amazonía & 2018 & $\mathrm{E}$ & $50+$ \\
\hline & \multirow{3}{*}{ V } & Cacao Ancestral & 2017 & $\mathrm{E}$ & $10+$ \\
\hline & & Guía Virtual de la Ciudad del Tena & 2017 & $\mathrm{E}$ & $50+$ \\
\hline & & Ruta Ancestral del Cacao & 2017 & $\mathrm{E}$ & $10+$ \\
\hline \multirow{3}{*}{ Orellana } & \multirow{3}{*}{$\mathrm{V}$} & Loreto Turístico - Guía Movil & 2018 & $\mathrm{E}, \mathrm{I}$ & $500+$ \\
\hline & & Orellana Turistica & 2017 & $\mathrm{E}$ & $500+$ \\
\hline & & Turismo Orellana & 2018 & $\mathrm{E}$ & $100+$ \\
\hline \multirow{3}{*}{ Pastaza } & $\mathrm{E}$ & Puyo Viajero & 2017 & $\mathrm{E}$ & $10+$ \\
\hline & \multirow{2}{*}{$\mathrm{V}$} & El Camino del Jaguar & 2018 & $\mathrm{E}$ & $5+$ \\
\hline & & Pastaza travel & 2018 & $\mathrm{E}$ & $10+$ \\
\hline \multirow{25}{*}{ Pichincha } & Ed & Museos Bellas Artes de Quito & 2018 & $\mathrm{E}$ & $10+$ \\
\hline & $\mathrm{E}$ & Quito App & 2018 & $\mathrm{E}$ & $10.000+$ \\
\hline & MyN & Mejia Travel & 2018 & $\mathrm{E}$ & $50+$ \\
\hline & \multirow{22}{*}{ V } & CarpeDM & 2017 & I & $5+$ \\
\hline & & CarpeDM Adventures & 2016 & I & $50+$ \\
\hline & & Cayambe Turístico & 2017 & $\mathrm{E}$ & $100+$ \\
\hline & & GOUIO & 2018 & E, I & $1000+$ \\
\hline & & Iglesias de Quito & 2016 & $\mathrm{E}$ & $100+$ \\
\hline & & La Mitad del Mundo Maps and Travel Guide & 2018 & $\mathrm{I}$ & $1+$ \\
\hline & & Mapa de Quito offline & 2018 & $\mathrm{E}$ & $1.000+$ \\
\hline & & Mejía & 2017 & $\mathrm{E}$ & $5+$ \\
\hline & & Mejia Tour & 2015 & $\mathrm{E}$ & $10+$ \\
\hline & & Mejía Tour Guía & 2018 & $\mathrm{E}$ & $10+$ \\
\hline & & Mindo & 2017 & $\mathrm{E}$ & $100+$ \\
\hline & & Mindo - Las Terrazas de Dana & 2015 & $\mathrm{I}$ & $50+$ \\
\hline & & Mitad del Mundo & 2018 & $\mathrm{E}, \mathrm{I}$ & $100+$ \\
\hline & & MovilizateUIO & 2018 & $\mathrm{E}$ & $10.000+$ \\
\hline & & Parques de Quito & 2014 & $\mathrm{E}$ & $100+$ \\
\hline & & Quito - Travel \& Explore & 2017 & I & $10+$ \\
\hline & & Quito City Guide & 2016 & $\mathrm{E}$ & $100+$ \\
\hline & & Quito Ecuador Map & 2017 & $\mathrm{I}$ & $1.000+$ \\
\hline & & Quito en tu Mano & 2016 & E, I, P, F, A, It & $5.000+$ \\
\hline & & Quito Guía Turística & 2017 & $\mathrm{E}$ & $10+$ \\
\hline & & Quito Guía Turística y mapa & 2018 & $\mathrm{E}$ & $1+$ \\
\hline & & Quito Guide \& Top Activites & 2013 & $\mathrm{I}$ & $100+$ \\
\hline
\end{tabular}




\begin{tabular}{|c|c|c|c|c|c|}
\hline Región & Cat $^{1}$ & Nombre & $\mathbf{A n ̃ o}^{2}$ & Idioma $^{3}$ & Descargas \\
\hline & & Quito Map & 2016 & E, I & $10+$ \\
\hline & & Quito Map and Walks & 2018 & I & $1000+$ \\
\hline & & Quito Mapp & 2016 & $\mathrm{E}, \mathrm{I}$ & $1000+$ \\
\hline & & Quito Offline Map Travel Guide & 2017 & $\mathrm{I}$ & $5+$ \\
\hline & & Quito Offline Travel Guide & 2017 & $\mathrm{I}$ & $1+$ \\
\hline & & Quito Tour Pass & 2015 & E, I & $100+$ \\
\hline & & Quito Walking Tour & 2018 & E, I & $100+$ \\
\hline & & Quito Wiki & 2018 & $\mathrm{I}$ & $1+$ \\
\hline & & Quito: Guía & 2018 & $\mathrm{E}, \mathrm{I}$ & $100+$ \\
\hline & & Tour Cantón Mejía & 2015 & $\mathrm{E}$ & $10+$ \\
\hline & $\mathrm{V}$ & Aeropuerto de Quito & 2017 & $\mathrm{E}$ & $1.000+$ \\
\hline Santa Elena & $\mathrm{V}$ & Santa Elena en tu Mano & 2016 & E, I, P, F, A, It & $1.000+$ \\
\hline \multirow{2}{*}{$\begin{array}{l}\text { Santo } \\
\text { Domingo }\end{array}$} & \multirow{2}{*}{$\mathrm{V}$} & Guía Santo Domingo Travel & 2016 & $\mathrm{E}$ & $1000+$ \\
\hline & & Santo Domingo OnLine & 2017 & $\mathrm{E}$ & $500+$ \\
\hline Carchi & $\mathrm{V}$ & Turismo Carchi & 2016 & $\mathrm{E}$ & $10+$ \\
\hline \multirow{5}{*}{ Tungurahua } & $\mathrm{C}$ & Tungurahua Tour & 2018 & $\mathrm{E}$ & $5+$ \\
\hline & \multirow{4}{*}{$\mathrm{V}$} & Baños de Agua Santa & 2018 & E, I & $10+$ \\
\hline & & Carturtungurahua & 2018 & $\mathrm{E}$ & $5+$ \\
\hline & & Samari SPA & 2018 & $\mathrm{E}$ & $10+$ \\
\hline & & Tungurahua en tu Mano & 2017 & $\mathrm{E}, \mathrm{I}, \mathrm{P}, \mathrm{F}, \mathrm{A}, \mathrm{It}$ & $500+$ \\
\hline
\end{tabular}

${ }^{1}$ MyN: Mapas y Navegación; NyR: Noticias y Revistas; V: Viajes y guías, E: Entretenimiento; H: Herramientas

${ }^{2}$ Año de actualización de la aplicación, considerando 2018 como año máximo de actualización.

${ }^{3}$ E: Español; I: Inglés; P: Portugués; F: Francés; It: Italiano; A: Alemán

${ }^{4}$ N.d.: No disponible

\subsection{Perfil del uso de aplicaciones por parte de los turistas que visitan el cantón Tena}

De los 382 turistas encuestados, el 57\% fueron mujeres, siendo su participación mayoritaria tanto entre nacionales como extranjeros. Resultado que concuerda con los resultados obtenidos por Carrera Miranda (2018), en su estudio para determinar el perfil del turista que visita la provincia de Napo. En cuanto a la edad de los encuestados, para su análisis se agruparon en tres generaciones etarias, observando que sobresale la Generación Y (Millennials), es decir, personas de 19 a 36 años (Figura 1), que se caracterizan por tener un mayor nivel de educación, sea bachillerato, superior/ universitario o se encuentran cursando un nivel de instrucción educativa, que cuentan con un salario básico o superior, buscan nuevas experiencias, y manejan con frecuencia las redes sociales para dar a conocer el lugar que se encuentran visitando o que visitaron. De acuerdo con Bauzá (2015) en su investigación sobre el nuevo segmento, los millennials en el turismo, el nivel de estudios es mayormente alto, ya que el $74 \%$ de los participantes han obtenido una carrera universitaria, el 14\% tiene la educación secundaria, el $8 \%$ posee un ciclo de formación profesional y, por último, el 2\% ha obtenido el bachillerato. También, es importante destacar de esta investigación la preferencia de los millennials con quien viaja, el 36,95\% viaja con amigos/conocidos, en pareja y familia ocupan el $21,74 \%$ y sólo $19,57 \%$. Así mismo, se pudo determinar que los millennials es el potencial mercado que estarían dispuestos a utilizar la App a proponer.

Las generaciones $\mathrm{X}$ y Z, aunque obtuvieron una mínima participación en la encuesta, no pueden ser descartados, debido a su potencialidad para el uso de Apps. La generación X se caracteriza por realizar viajes familiares, gozan de un salario, se encuentran próximos a jubilarse. En cambio, la generación Z, que aún se encuentra en surgimiento, se caracteriza por disponer de la tecnología en su mano y estar constantemente en uso, aun son dependientes de sus padres, realizan viajes con la familia o viajes escolares.

Desde que los Smartphone llegaron al mercado, el uso de Apps fue creciendo a gran medida. En la actualidad, la mayoría de las personas están familiarizados con el uso de alguna de ellas.

Los resultados obtenidos en la presente investigación han determinado que el turista que visita el cantón Tena sea nacional o extranjero, dentro de sus preferencias de Apps, el uso de redes sociales ocupa el primer lugar (Figura 2). Según Google, los viajeros sienten el impulso de compartir las experiencias surgidas en sus viajes en las redes sociales, es decir el 92\% lo hacen (Hosteltur, 2017). 
Figura 1: Distribución de los encuestados por generación etárea

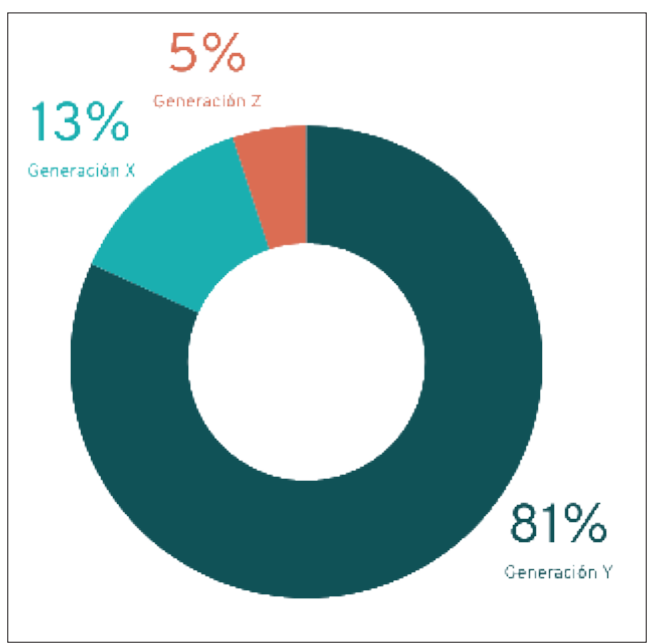

Del conjunto de Apps que conforma las redes sociales, Facebook es la más utilizada, seguida por aquellas que facilitan la Comunicación (principalmente enviar y recibir correos electrónicos) con un $21 \%$. El portal digital Statista coloca a esta categoría en la segunda posición de la estadística general con un 99,4\% de alcance en el mercado mundial de las Apps más populares de Android (Statista, 2017). Además, de acuerdo con el Observatorio TIC del MINTEL (2018b), a nivel nacional el uso de correos electrónicos en dispositivos móviles ocupa un 83,66\%. También cabe destacar que estas herramientas han mejorado y en la actualidad permiten almacenar documentos, archivos, realizar formularios, etc. Las categorías Noticias/Entretenimiento y Viajes ocupan el tercer y cuarto lugar, respectivamente. Finalmente, las categorías relacionadas con Libros, Juegos, Ejercicios, Shopping/E-commerce posee un $12-19 \%$ de preferencia de uso.

Con los datos obtenidos se pueden determinar estrategias para promocionar la App, mediante el uso de estas herramientas digitales y así llegar en mayor cantidad y calidad al turista que visita el cantón y sobre todo adquirir más mercado para el uso de las Apps turísticas.

\section{Figura 2: Preferencias de Apps por los turistas}

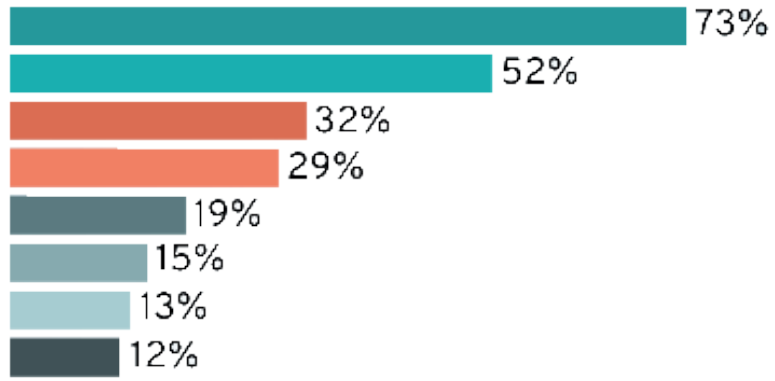

Sacial Media

Comunicacion

Noticias/Entretenimiento

Apps de Turismo

Libras

Juegas

Ejercicio:

Shopping 
En la actualidad, las herramientas digitales han superado a los medios tradicionales para adquirir información. Los sitios web ocupan el primer lugar como principal herramienta de información digital, de acuerdo con Castro Ricalde et al. (2018) a partir de la última década del siglo XX hasta hoy en día, el uso de la web en el turismo ha permitido que el acceso a la información sea más rápida y eficiente, lo que está provocando importantes cambios en materia de infraestructura de telecomunicaciones, lo que permite y facilita la promoción y comercialización de atractivos turísticos en todo momento y en cualquier parte del universo. Luna Cortés \& Berenguer Contri (2013) destacan el aspecto funcional de la información, ya que consideran que la información en destino debe ser lo más simple y directa posible, con el objetivo de aclarar dudas e inquietudes de los turistas de manera rápida y sencilla, situación que influye directamente en la elección de la fuente y la intensidad de la búsqueda de información. También, es importante mencionar que en la actualidad la elaboración de un sitio web es muy sencilla a través de distintas plataformas, las cuales permiten que personas con conocimientos informáticos de programación o codificación bajos pueda diseñar una página web o un blog. Es así como, aproximadamente 7 de cada 10 encuestados señalaron que los sitios web son su principal medio de obtención de información (Figura 3).

\section{Figura 3: Medios de información utilizados por los turistas}

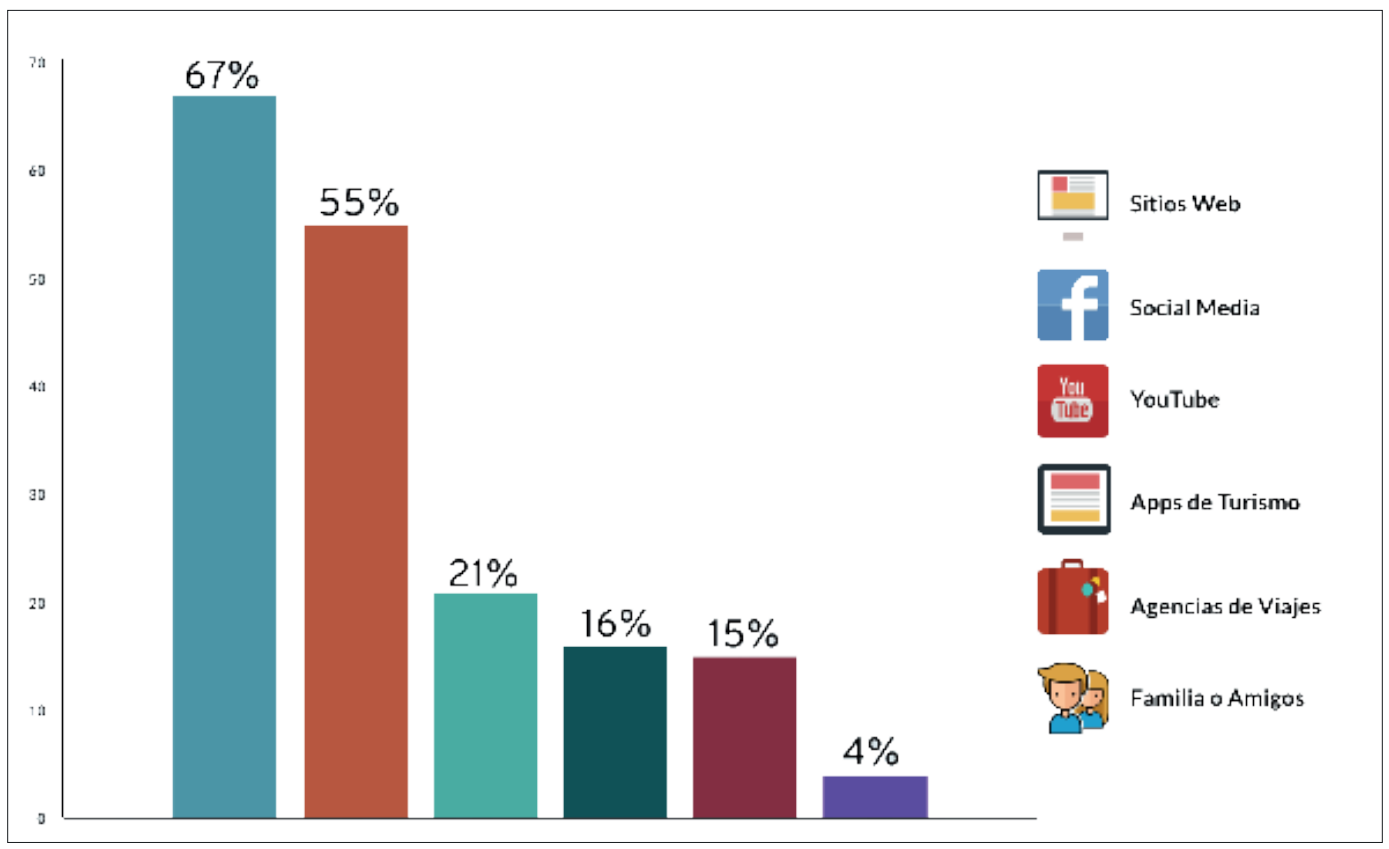

En segundo lugar, de preferencia se identificó a las redes sociales, utilizadas por aproximadamente la mitad de los encuestados. Hosteltur (2017) menciona que 1 de cada 5 viajeros antes de planificar su viaje utiliza las redes sociales como fuente de inspiración para escoger su futuro destino, que durante el viaje siguen conectados a las redes sociales y se estima que el $72 \%$ de las personas publican fotos de su viaje. Otra de las herramientas digitales que está adquiriendo mayor importancia por parte de viajeros es Youtube, donde bloggers de todo el mundo graban su experiencia en cada destino que visitan y además dan recomendaciones, consejos y recomendaciones para los que se animan a visitar algún destino que ellos ya lo hicieron. En tercer y cuarto lugar de preferencia se señalaron a las Apps de turismo y Agencias de Viajes, respectivamente. Estos niveles de preferencia ponen de manifiesto la importancia que tiene cada vez más el uso de medios digitales para planificar, disfrutar y compartir un viaje. Recomendaciones de familia y amigos cada vez tiene menor importancia en la selección de destinos. 
En cuanto al uso de Apps turísticas, solo cuatro de cada 10 turistas, tanto nacionales como extranjeros, las utilizan en sus viajes (Figura 4). La principal App utilizada es Google Maps (29\%), debido a los servicios de georreferenciación que presta. Otras Apps como Maps.me y Waze ocupan un 11\%. En referencia a reservaciones de hoteles e información turística, TripAdvisor y Booking son las Apps preferidas. Finalmente, las Apps de información turística como: Lonely Planet, Hostel World, Moovit, AirBnB, Izl.travel y más, agrupadas obtuvieron un $24 \%$ de uso. Grevtsova (2016) destaca la importancia de la gamificación mediante la generación de estrategias lúdicas e interactivas, lo que haría más atractivas las TICs en general a los turistas y visitantes, generando una experiencia de calidad y única. Esto permitiría que las apps relacionadas con información turística, patrimonio, eventos, etc., sean más atractivas y su uso aumente. Ya que se observa que apps relacionadas con movilidad y reservas son las más utilizadas al visitar el Tena.

\section{Figura 4: Uso de Apps de turismo}

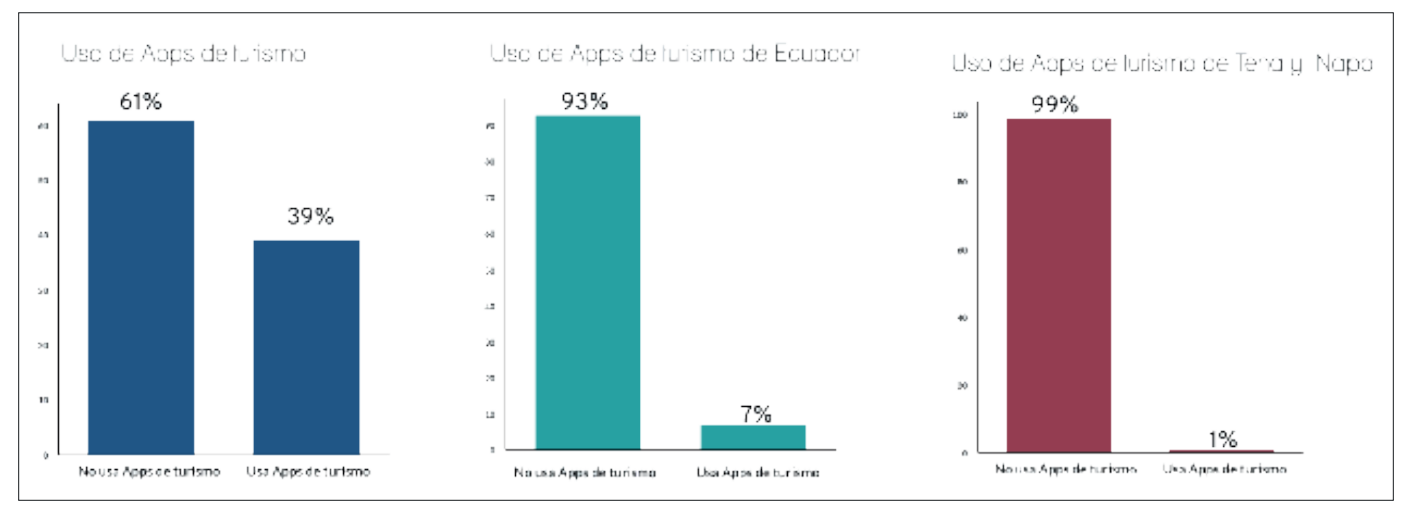

Se determinó que solo 1 de cada 10 de los turistas encuestados usan o conocen alguna App de turismo de Ecuador. De las respuestas obtenidas, el 50\% correspondieron a Apps con información turística general de Ecuador como: Travel Ecuador, Big Guide Ecuador, Discover Ecuador \& More, y GoRaymi. El 40\% representa a Apps de turismo específicamente de Quito como: Quito Turismo, Quito App, GOUIO. Por último, con un 10\%, otras Apps utilizadas son: Puyo Travel y Turismo Fácil. Como aspecto negativo se constató que la gran mayoría no usa o desconoce las Apps que están disponibles a nivel nacional. Finalmente, la mayoría de los turistas encuestados desconocen de la existencia de las cuatro Apps turísticas de Tena, situación que da a entender el mínimo interés de promocionar estas herramientas por parte de las entidades encargadas de las mismas. Así como también se pone de manifiesto un gasto significativo en la inversión de estas herramientas que no ha sido bien aprovechado, ya que diseñar y poner en funcionamiento una App tiene un costo aproximado de 4.500 a 20.000 USD, de acuerdo con su complejidad.

Sobre los sistemas operativos utilizados, el 79\% de los turistas encuestados utilizan Android, seguido por el 19\% que utiliza IOS Apple y el 2\% Windows Phone. Esta gran diferencia se explica porque en el 2016, el $84,8 \%$ de los teléfonos móviles vendidos en todo el mundo contaban con un sistema Android (Moreno, 2017).

\subsection{Diagnóstico del uso de TICs desde los prestadores de servicio de Tena}

El uso de nuevas tecnologías en el sector del turismo ha sido una herramienta clave para mejorar los servicios o promocionar un destino turístico. En el caso del cantón Tena, el presente diagnóstico da una perspectiva sobre las herramientas digitales que utilizan los prestadores de servicios turísticos para darse a conocer en el mundo digital. Toda la información presentada en este apartado corresponde a la sistematización de las opiniones y comentarios de 140 prestadores de servicios del cantón Tena, recogidos a través de las entrevistas realizadas.

El 85\% utiliza alguna herramienta digital para dar a conocer los servicios y ofertas que disponen (Figura 5), de las cuales las redes sociales son las más utilizadas, destacando la red social Facebook (63\% de aceptación). Referente al uso de página web, de los 140 establecimientos consultados, solo el 23\% 
dispone de una, principalmente alojamientos. De igual manera, los alojamientos utilizan herramientas digitales para su gestión de reservas, principalmente Booking y TripAdvisor. Es decir, los prestadores de servicios se han enfocado principalmente en la gestión de reservas, pero no existe un verdadero ejercicio de entender el mercado o las nuevas tendencias y demandas, aspectos que son considerados como ventajas competitivas, especialmente de las pymes (López Rodríguez \& López Rodríguez, 2018; Ruiz Gómez et al., 2016). Así como tampoco, se evidencia un trabajo hacia el fortalecimiento de la competitividad y consolidación del destino turístico basado en las TICs, las cuales son un fuerte catalizador de este proceso (Bethapudi, 2013; Lemos Gomes et al., 2018). Aunque Jacobsen \& Munar (2012) identificaron que en destinos bien conocidos las redes sociales y medios digitales tienen menor relevancia. También, producto de la investigación se observó que la mayoría de los prestadores de servicios turísticos no manejan un plan de marketing para potencializar su marca, consolidar su segmento de mercado o generar ventas. Algunos de los prestadores disponían de más de una cuenta en Facebook que en su mayoría no disponía de información, demostrando un poco interés de manejo de la misma.

En relación con el pago por servicios digitales, el 39\% de los prestadores de servicios entrevistados pagan por pertenecer a un servicio, o contratan alguna promoción. El valor de pago varía en un rango entre 10 y 600 USD, que depende del tiempo y el alcance de la promoción ofertada. Por ejemplo: por realizar promoción por Facebook pagan un monto de 30 USD por un lapso de siete días, en el caso del dominio para páginas web pagan alrededor de 90 USD anuales, en algunos casos han pagado por un paquete completo, es decir por página web 200 USD, en Facebook 120 USD y por un Software 150 USD. Otros tipos de pago, como por ejemplo por comisión, en el caso de Booking se paga el $2 \%$ por cada pax. Para contrastar, el $35 \%$ no pagan por el servicio digital, sin embargo, utilizan una cuenta por Facebook o WhatsApp para realizar reservaciones.

\section{Figura 5: Uso de herramientas digitales}

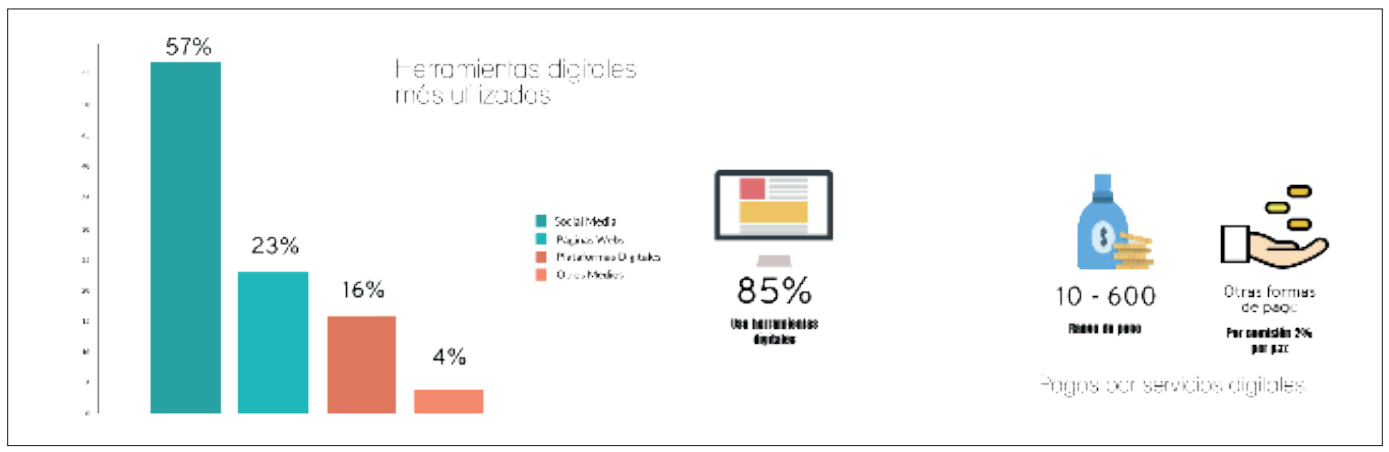

Los prestadores de servicio conocen o han utilizado una App relacionado a turismo en un 31\%, y en este caso Booking fue la App más conocida (Figura 6). Este resultado destaca la falta de interés o conocimiento de la aplicación de las TICs en el ámbito turístico, situación que no permite aprovechar los impactos positivos que tienen el desarrollo de la actividad, en especial en subsectores como alojamiento y restauración (Aramendia-Muneta \& Ollo-López, 2013). Así también, cabe recordar que la provincia de Napo y el cantón Tena disponen de cuatro Apps de turismo en las plataformas de Play Store y App Store, y que sólo el 6\% de los prestadores de servicios las conocen, es decir, 8 de los 140 prestadores de servicios entrevistados, poniendo de manifiesto un desconocimiento significativo de estas herramientas de promoción turística locales. 
Figura 6. Apps de turismo: conocimiento y uso
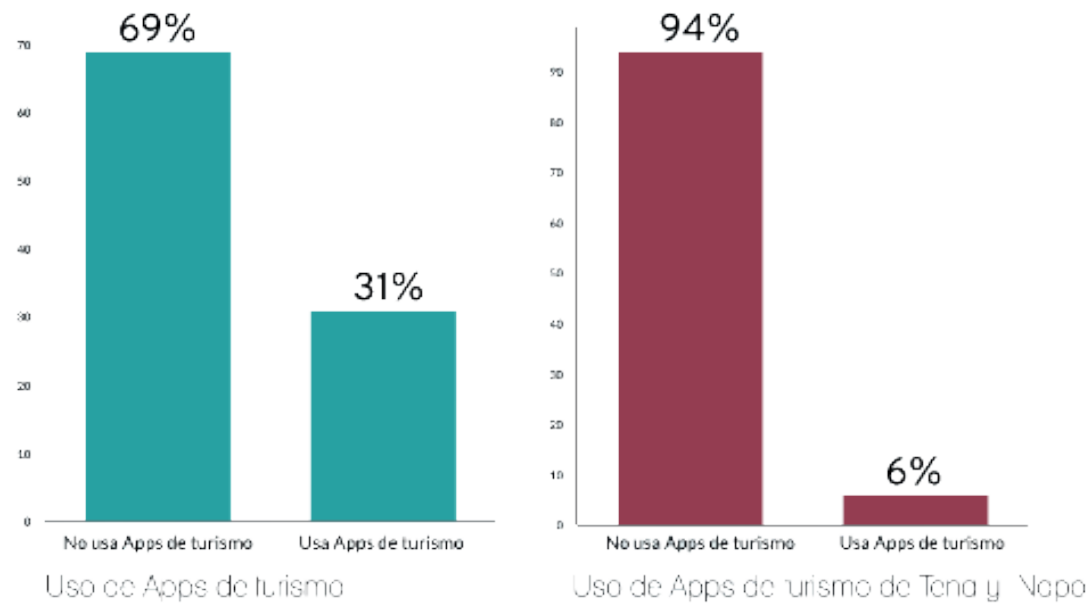

Los datos relacionados con el uso de Apps concuerdan con lo señalado por Cerezo Medina \& Guevara Plaza (2015), quienes indican que las TICs son consideradas relevantes en el sector turístico, pero que lastimosamente esa percepción no se traduce en equipamiento tecnológico ni en la implementación de acciones formativas dentro de las empresas turísticas. Es decir, existen recursos, pero los prestadores de servicio en el Tena han trabajado únicamente con Apps bastante conocidas para reservas, mas no para proveer información completa a los posibles turistas.

Por otra parte, es importante que los prestadores de servicio se capaciten en el diseño de imagen y contenidos, como lo recomiendan Ukpabi \& Karjaluoto (2017), para mejorar la comunicación con los turistas. También en cultural empresaria basada en TICs, para mejorar las comunicaciones dentro de las empresas, implementar proceso para una mejor gestión de personal, inventarios, etc. (Farkhondehzadeh et al., 2013). Otro tema de capacitación recomendable es la medición del tráfico de sus sitios webs para identificar el comportamiento y las tendencias de los clientes, así como también analizar la competencia (precios y políticas de precios), aspectos que pueden ser útiles para que las empresas turísticas actúen en consecuencia y mejoren la comunicación con los principales actores relacionados (Ruiz-Molina et al., 2013). Así como que los gobiernos locales, como contraparte, trabajen en temas de conectividad, de manera que los prestadores de servicios puedan ofrecer servicios mediante plataformas con una mayor velocidad, y que los turistas puedan acceder a estos servicios con una mayor cobertura (Bethapudi, 2013).

\section{Conclusiones}

En Ecuador se han desarrollado y registrado un total de $150 \mathrm{Apps}$, de acuerdo con la plataforma Google Play, siendo Pichincha (Quito) una de las provincias con mayor desarrollo de Apps. Con relación a la situación del cantón Tena, se identificaron un total de cuatro Apps de turismo registradas para promocionar el cantón y la provincia. Lastimosamente estas aplicaciones son ampliamente desconocidas tanto por turistas como por prestadores de servicios. Este desconocimiento pone de manifiesto la falta de una cultura digital por parte de los prestadores de servicios y las autoridades locales, quienes aún no son conscientes de los beneficios que conlleva la adopción de nuevas tecnologías para el sector turístico. Por tanto, urge que el sector turístico privado trabaje hacia digitalizar la oferta turística del cantón, de manera que las empresas turísticas locales puedan beneficiarse de una mayor publicidad, una mayor comunicación con sus clientes y establezcan canales propios de comercialización.

Por otra parte, el sector público (gobiernos municipal y provincial), dado que posee las competencias en materia de turismo en el territorio, puede apoyar estos procesos de digitalización al fortalecer la promoción del destino a través de los distintos medios digitales, y al mejorar la infraestructura de conectividad digital. 
El turista que visita el cantón Tena prefiere el uso de redes sociales durante sus viajes, seguido de Apps de Comunicación y de Apps Noticias y Entretenimiento. El medio más utilizado por el turista para informarse del lugar que desea visitar son sitios web. Similar sucede con los prestadores de servicios, quienes prefieren las redes sociales para promocionar y comercializar sus establecimientos, siendo Facebook la red social más utilizada. Estos resultados son similares a los identificados en investigaciones previas, siendo un reto para los prestadores de servicios el generar contenidos más dinámicos, lúdicos y que generen, o incentiven, nuevas experiencias turísticas. Para ello, es importante que desde los distintos gestores del sector turístico, tanto públicos como privados, se fortalezca el uso de redes sociales a través de capacitaciones.

Sin duda, las generaciones y la tecnología van de la mano, ya que da a entender cómo cada una de ellas se desarrollaron o se encuentran en surgimiento, esto también determina su comportamiento al momento de realizar un viaje. En la actualidad, en Ecuador aún no existe una investigación a profundidad sobre el perfil del turista de acuerdo con su generación, cuáles son sus preferencias en tecnología, qué medios digitales utilizan para informarse de un destino, su necesidad de estar conectado en un destino o sitio de visita. Por lo cual, este trabajo da una proyección para nuevas investigaciones y determinar el nuevo turista es decir el "Turista Digital”, de manera que se aporte a la planificación de destinos turísticos inteligentes.

\section{Bibliografía}

Albacete Sáez, C. A., \& Herrera Fernández, M. 2012. Las Tecnologías de la Información y Comunicación en el Sector Turístico. Una aproximación al uso de las redes sociales. Centro de Estudios Ramón Areces, S.A.

Álvarez, R. B., \& Mayo, I. C. 2009. Las tecnologías de la información y la comunicación en la educación superior. Estudio descriptivo y de revisión. Revista Iberoamericana de Educación, 50(7), 1-12. https:// doi.org/10.35362/RIE5071965

Andalucía es digital. 2019. Turismo y Nuevas Tecnologías: Tendencias 2019 y Recursos TIC para el Impulso de un sector clave. https://www.blog.andaluciaesdigital.es/turismo-y-nuevas-tecnologias/

Andrade Suárez, J. M. 2012. El uso de Internet como fuente de información turística: propuesta metodológica para el análisis de su importancia en la creación de la imagen del destino. Papers de Turisme, 52, 44-62. http://www.papersdeturisme.gva.es/ojs/index.php/Papers/article/view/63

Aplicación Annie. 2016. Ingresos mundiales de la tienda de aplicaciones móviles en 2015, 2016 y 2020 (en miles de millones de dólares estadounidenses). https://www.statista.com/statistics/220186/total-global-in-app-revenue-forecast/

Aplicación Annie. 2018. Número de descargas de aplicaciones móviles en todo el mundo en 2017, 2018 y 2022 (en miles de millones). https://www.statista.com/statistics/271644/worldwide-free-and-paid-mobile-app-store-downloads/

Aramendia-Muneta, M. E., \& Ollo-López, A. 2013. ICT Impact on tourism industry. International Journal of Management Cases, 15(2), 84-98. http://www.ijmc.org/IJMC/Vol_15.2_files/15.2.pdf\#page=87

Bauzá, M. 2015. Turismo del Siglo XXI, análisis de un nuevo segmento, los Millennials. Universitat de les Illes Balears.

Bethapudi, A. 2013. the Role of Ict in Tourism Industry. Journal of Applied Economics and Business, 1(4), 67-79. http://www.aebjournal.org/articles/0104/010406.pdf

Blanco, J. 2015. Libro Blanco de los Destinos Turísticos Inteligentes. Estrategias y soluciones para fomentar la innovación en el turimo digital. LID Editorial Empresarial.

Cámpora Espí, E. 2013. Estudio del impacto de las TIC en el turismo: análisis de su influencia en los habitantes de la ciudad de Gandía durante la planificación de un viaje. Universidad Politecnica de Valencia.

Carrera Miranda, A. 2018. Estudio del comportamiento de la demanda turística nacional e internacional en la Provincia de Napo. Universidad Central del Ecuador.

Castro Ricalde, D., Peñaloza Suárez, L., \& Tamayo Salcedo, A. L. 2018. Tecnologías en línea populares para viajar: ¿cuáles utilizan los jóvenes universitarios para hacer turismo? Actualidades Investigativas en Educación, 18(2). https://doi.org/10.15517/aie.v18i2.33149

Cerezo Medina, A., \& Guevara Plaza, A. 2015. Necesidades formativas tecnológicas en turismo. Estudio de caso de Andalucía. PASOS Revista de turismo y patrimonio cultural, 13(4), 913-929.

Clement, J. 2020. Number of mobile app downloads worldwide from 2016 to 2019. Statista. https://www. statista.com/statistics/271644/worldwide-free-and-paid-mobile-app-store-downloads/ 
Farkhondehzadeh, A., Robat Karim, M. R., Roshanfekr, M., Azizi, J., \& Legha Hatami, F. 2013. E-Tourism: The role of ICT in tourism industry. European Online Journal of Natural and Social Sciences, 2(3), 566-573.

Florido-Benítez, L., Del Alcázar Martínez, B., \& González Robles, E. M. 2015. El beneficio de la gestión de relación entre las empresas y turistas a través de las aplicaciones móviles como herramienta de marketing y elemento diferenciador de los destinos turísticos. Turismo y Desarrollo Local, 5(2), 57-69.

GADM Tena. 2017a. Catastro Turístico del Cantón Tena 2017.

GADM Tena. 2017b. Consolidado de Afluencia de Turistas en el Cantón Tena 2006 - 2017.

Grevtsova, I. 2016. Tendencias del uso de las tecnologías móviles en espacios urbanos: m-learning y patrimonio cultural. Revista PH 90, 90, 132-151. https://doi.org/10.33349/2016.0.3695

Hosteltur. 2017. El verdadero sentido de las Redes Sociales en Turismo. 12 de junio. https://www.hosteltur. com/comunidad/005575_el-verdadero-sentido-de-las-redes-sociales-en-turismo.html

Jacobsen, J. K. S., \& Munar, A. M. 2012. Tourist information search and destination choice in a digital age. Tourism Management Perspectives, 1(1), 39-47. https://doi.org/10.1016/j.tmp.2011.12.005

Januszewska, M., Jaremen, D., \& Nawrocka, E. 2015. The effects of the use of ICT by tourism enterprises. Zeszyty Naukowe Uniwersytetu Szczecińskiego. Service Management, 16(883), 65-73. https://doi. org/10.18276/smt.2015.16-07

Lemos Gomes, E., Henrique Moscard, E., Alves Pinto, M. J., \& Nakatani, M. S. M. 2018. Las relaciones entre la información turística y las tecnologías de la información y la comunicación. Análisis de publicaciones científicas en revistas latinoamericanas. Estudios y Perspectivas en Turismo, 27(3), $569-587$.

López Rodríguez, A. L., \& López Rodríguez, S. A. 2018. Impacto de las TIC en el turismo: Caso colombiano. Cuadernos de Turismo, 41, 399-418. https://doi.org/http://dx.doi.org/10.6018/turismo.41.327081

Luna Cortés, G., \& Berenguer Contri, G. 2013. La búsqueda y el valor de la información en los destinos turísticos. Papers de Turisme, 523, 18-43. http://www.papersdeturisme.gva.es/ojs/index.php/Papers/ article/view/126

Maschini, S. 2012. Claves del Marketing Digital. La nueva comunicación empresarial en el mundo 3.0. Vanguardia Ediciones, S.L.

MINTEL. 2018a. Libro Blanco de la Sociedad de la Información y del Conocimiento.

MINTEL. 2018b. Observatorio TIC. https://observatoriotic.mintel.gob.ec/estadistica/

MINTEL. 2019. Indicadores y Estadistica. https://www.telecomunicaciones.gob.ec/estadistica/index.html

Molano, A., Álvarez, E., Urrego, J., Herrera, C., \& González, M. 2014. Generaciones y tecnologías. Corporación Digital Colombia.

Moreno, G. 2017. Android e iOS dominan más del 99\% del mercado. Statista. es.statista.com/grafico/8203/ android-e-ios-dominan-mas-del-99-del-mercado/

Muñoz Prieto, M., Fragueiro Barreiro, M., \& Ayuso Manso, M. 2013. La importancia de las redes sociales en el ámbito educativo. Escuela abierta: Revista de investigación educativa, 16, 91-104.

Muñoz, R. 2018. El número de líneas móviles supera por primera vez a la población mundial. Los usuarios alcanzaron los 5.000 millones en 2017, pero las tarjetas SIM se elevaron a 7.800 millones. El País.

Ruiz-Molina, M. E., Gil-Saura, I., \& Šerić, M. 2013. The use of ICT in established and emerging tourist destinations: A comparative analysis in hotels. Journal of Hospitality and Tourism Technology, 4(2), 96-118. https://doi.org/10.1108/JHTT-Oct-2011-0033

Ruiz Gómez, L. M., Rodríguez Fernández, L., \& Muñoz Martínez, C. 2016. Aplicación de tecnologías de la comunicación en el sector turístico español. Boletín Económico de ICE, 3080, 59-65. https://doi. org/10.32796/bice.2016.3080.5596

Sánchez Jiménez, M. Á., \& Ravina Ripoll, R. 2017. Análisis de las aplicaciones móviles de destinos turísticos y su accesabilidad. Teoría y Praxis, 15(31), 3-26. https://doi.org/10.5377/typ.v0i31.6379

Sanz Blas, S., Martí Parreño, J., \& Ruiz Mafé, C. 2012. Aplicaciones publicitarias para móvil: conocimiento, actitudes, motivos de uso y valoración por parte de los adolescentes españoles. Pensar la Publicidad. Revista Internacional de Investigaciones Publicitarias, 6(1), 255-270. https://doi. org/10.5209/rev_pepu.2012.v6.n1.38665

Segovia Borray, C. E., Bermeo Andrade, H. P., \& González Bañales, D. L. 2014. La integración de las tecnologías de la información y la comunicación (TIC) como estrategia para generar competitividad en el sector turismo: una investigación en la región de Tolima (Colombia). Universidad \& Empresa, 16(27), 137-156. https://doi.org/10.12804/rev.univ.empresa.27.2014.05 
Ukpabi, D. C., \& Karjaluoto, H. 2017. Consumers' acceptance of information and communications technology in tourism: A review. Telematics and Informatics, 34(5), 618-644. https://doi.org/10.1016/j. tele.2016.12.002

UNWTO. 2018a. El Turismo y la Transformación Digital. Organización Mundial del Turismo.

UNWTO. 2018b. Turismo en la Era Digital. Organización Mundial del Turismo. DMT \& Innovación. http://wtd.unwto.org/es/content/dmt-2018-la-cuarta-revolucion-industrial

Urvina Alejandro, M. A. 2019. Propuesta de una aplicación móvil celular turística para el cantón Tena. Universidad Central del Ecuador.

Vieira Soares, A. L., Mendes-Filho, L., \& do Nascimento Barbosa Cacho, A. 2017. Evaluación de la Información de una Aplicacion Turística. Un análisis realizado por profesionales del turismo sobre la e-Guía Find Natal (Brasil). Estudios y Prespectivas en Turismo, 26(4), 884-904. 